\title{
Introduction to special topic issue on technology in neurorehabilitation
}

\author{
Michael Rosen \\ Guest Editor
}

Technology can serve several purposes in the lives of people with neurological disabilities - augmenting their treatment and enhancing everyday living. It can be assistive, supplementing or supporting an individual in daily activities in a way which compensates, at least in part, for abilities which have been diminished by the effects of atypical development, injury or disease. Technology may play a therapeutic role as an alternative or complement to drug treatment, physical and occupational therapies and other conventional modalities. And some technology is evaluative, providing sensitive objective means for exposing the mechanism of a disability and assessing its severity and characteristics for clinical or research purposes.

The articles in this special topic issue of NeuroRehabilitation span all of these categories, often in combination. Augmentative communication devices, as described in the article by Beth Mineo-Mollica, are primarily assistive, although their use by individuals with developmental disabilities may help advance language and social skills. The same can be said for cognitive prosthetics, i.e., computer programs custom-designed to prompt and support functional activities. The article by Elliot Cole on this topic reports surprising improvements in aspects of cognitive function in some cases where only support was intended. Will Durfee's report on functional electrical stimulation describes, among others, applications to stroke patients which are assistive or therapeutic, offering both electro-exercise for increasing strength, and limited control of functional grasp and gait. Home health care technologies, described by Corinna Lathan, are meant to provide convenient assessment of vital health parameters as well as self- or family-administered home treatment. Virtual reality applications in cognitive rehab are almost all in the research domain, at present, but Cheryl Trepagnier's paper on this topic suggests the promise of these innovative technologies for both assessment and reme- diation of syndromes such as impaired face recognition characteristic of people with autism. Telerehabilitation is defined in my paper as a battery of techniques which employ telecommunications and information technologies to deliver therapies, monitoring, assessment and coaching at a distance.

The topics in technology covered in this issue were chosen to illustrate all three of these application categories. They also intentionally span topics from operational to conjectural - e.g., augmentative communication to virtual reality - and from mass-market high tech applied to neurorehab (computers as cognitive prostheses, for example) to highly specialized engineering for intimate interaction with human physiology (such as FES). In retrospect, however, some other commonalities and contrasts emerge to which I will call attention briefly as an introduction to this issue.

While the role of modern computing technology is obvious in some of these technologies and hidden in others, it is, in fact, essential to all of them. The seamless movement of dynamic images in virtual reality displays, ideally rapid enough that delays in response to user inputs are undetectable, demands application of computers which are substantially more powerful and more expensive than garden variety PC's. Such computing power demanded room-sized facilities until less than a decade ago and was completely unavailable another decade back. Electronic augmentative communication products ceased to be built from fixed-purposed circuits twenty years ago when the microelectronics revolution made it possible to obtain more function and infinite flexibility of function in much less space for less money by writing programs for single-chip computers. Functional electrical stimulation to overcome foot drop in individuals with strokes was first accomplished in the early sixties without computers. But coordinated stimulation of multiple muscles - often using implanted electronics, integrating signals from numerous feedback sensors, and obeying control laws which learn how to optimize the quality of limb movement is a practical impossibility without the miniaturization and flexibility provided by microcomputers. 
Engineering sophistication is reflected in all of the systems reviewed in this issue. Synthetic speech as output from AAC devices, radical compression of information for transmission over phone lines in telerehabilitation, body chemistry analysis in small boxes for home health monitoring, libraries-worth of memory electronically accessed in updating computer graphic images in virtual reality systems - these are just some examples. For most intended users, however, high technology per $s e$ has an effect which is somewhere between neutral and negative on the appeal of a product. What matters is the enhancement of function obtained and what cost the user must pay in terms of physical effort and mental load. This is almost a truism for product design in general, but the presence of disability magnifies its importance. The rules of good human factors design may be - and often are - violated for able-bodied consumers who have substantial reserves of adaptive ability and health. In contrast, a communication aid which requires learning several levels of operational rules and lacks clear feedback of its current mode may be completely beyond the use of an individual with impairment of working memory. Technology for individuals with disabilities should be predictable to the point of becoming transparent, providing an augmenting functional medium rather than a challenging new task. Home health technology which prompts use of medication or detects life-threatening changes in vital signs must be virtually flawless with regard to its interaction with the patient and care giver since the cost of human factors design errors could be fatalities.

It should be noted that functional transparency is not a sensible goal for prosthetic software which supports cognitive function. The computer program functions as a de facto personal assistant for a limited scope of activities (e.g., cooking tasks) and cannot vanish. It should, however, be a clear and unambiguous aid which is well tuned to the personal needs and abilities of its client. Further, the "mechanics" of using the computer which hosts the assistive software must be simple, free of contingent rules, and uncrashable. PC users familiar with the glitches common in the current best-selling graphical operating system will understand how unacceptable the standard freeze-ups, indecipherable error messages, and defaults to the more primitive underlying system command language would be for individuals who depend on the computer to decrease the cognitive demand of everyday tasks.

Another distinctly non-technical issue for the success of some of the more conjectural technologies described in this issue - for example therapy delivered by telehealth means, or virtual environments for treatment of aphasia - is cost and reimbursement. There is no incentive for commercial development of these approaches unless there is a clear sign that at least some insurers, managed care organizations, Medicare and/or Medicaid will pay therapists, neurologists and other physicians to make use of them. In the case of some potentially less expensive home health technologies, for example therapeutic exercise systems or vital sign monitoring devices, it may make sense to market products at prices which are within reach of families' own resources.

Along with reimbursement, competent and widespread application of these evaluative, therapeutic and assistive techniques requires rehabilitation professionals with the necessary training. Even for the better established technologies covered in this article, preservice curricula still commonly provide less than the necessary background. Graduate Speech-Language Pathologists, for example, do not all receive the education they will need in the strategies and products of AAC. In part, this reflects the (relative) newness of $\mathrm{AAC}$ technology and the proliferation of devices and techniques which makes it challenging for curricula to keep up. In my experience, it may also be indicative of a negative bias with regard to technology. Many physician and non-physician rehabilitation practitioners were drawn to their professions by their humanistic appeal and by the prospect of direct interaction with patients. For some, a corollary is that rehabilitation "hardware" is unappealing and at best a necessary evil. Therapeutic technology may be viewed as second best to hands-on. Objective instrumentation for assessment of function may be seen as providing only confirmation of what can already be detected by eye - or details which have little implication for treatment. Assistive technology is viewed by some as a last resort which interferes with efforts to return "natural" function and constitutes an admission of failure. And perhaps most of all, the rapid advances in the world of computing and other "high" technologies are daunting even to avid users and may be intimidating in the extreme to untrained would-be users. While these views may be exaggerated here and probably do not reflect the attitudes of a majority of rehabilitation professionals, they do pose a potential obstacle to application of powerful new approaches. Clinical "lead customers", academic physicians and therapists, and technology developers must take on the need for advanced training as an urgent joint venture. 